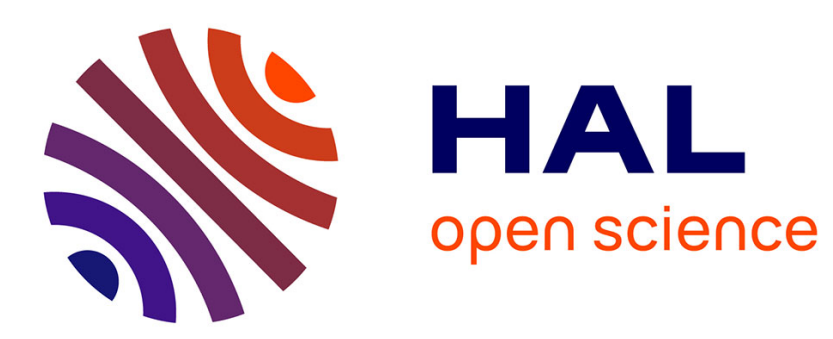

\title{
Patterning of a drying emulsion film
}

Aurore Bournigault-Nuquet, Sandrine Couderc, Jérôme Bibette, Jean Baudry

\section{To cite this version:}

Aurore Bournigault-Nuquet, Sandrine Couderc, Jérôme Bibette, Jean Baudry. Patterning of a drying emulsion film. Langmuir, 2021, 37 (30), pp.8924-8928. 10.1021/acs.langmuir.1c00246 . hal-03386282

\section{HAL Id: hal-03386282 \\ https://hal.science/hal-03386282}

Submitted on 19 Oct 2021

HAL is a multi-disciplinary open access archive for the deposit and dissemination of scientific research documents, whether they are published or not. The documents may come from teaching and research institutions in France or abroad, or from public or private research centers.
L'archive ouverte pluridisciplinaire HAL, est destinée au dépôt et à la diffusion de documents scientifiques de niveau recherche, publiés ou non, émanant des établissements d'enseignement et de recherche français ou étrangers, des laboratoires publics ou privés. 


\title{
Patterning of a drying emulsion film
}

\author{
Aurore Bournigault-Nuquet ${ }^{1,2}$, Sandrine Couderc², Jérôme Bibette ${ }^{1}$, Jean Baudry ${ }^{1 *}$ \\ 'Laboratoire Colloïdes et Matériaux Divisés, CBI, ESPCI Paris, Université PSL, CNRS, 75005 Paris, France \\ ${ }^{2}$ CHANEL Parfums Beauté, 8 rue du Cheval Blanc, Pantin, France \\ KEYWORDS Thin films, emulsions, drying
}

\begin{abstract}
Stabilizing layers of colloidal dispersions or emulsions to obtain homogeneous films is a real challenge. We describe here a new kind of instability in drying films of emulsions: during evaporation of the internal phase, cracks appear between the droplets, that create aggregates according to a regular pattern. We show that this pattern only appears if the emulsion is adhesive, i.e. if droplets stick together. The pattern exhibits a characteristic length which depends on adhesion strength and film thickness. These experimental results support a model where this instability is due to the gel structure and elastic properties of adhesive emulsions. Understanding this phenomenon will allow us to get a homogeneous film or to control it to get structured materials.
\end{abstract}

\section{INTRODUCTION}

Study of films is important for many different industrial domains such as coatings ${ }^{1}$, cosmetics ${ }^{2}$ and micro-electronics3. Deposited materials are usually particle dispersions as in paints or emulsions as in cosmetics, enabling to meet requirements such as anti-corrosion ${ }^{4}$, color ${ }^{5}$, sensoriality ${ }^{6}$. In addition, volatile phases are essential so that the material is fluid enough to be spread, but solid when dry. The evaporation, most of the time of part of the external phase, may provoke instabilities ${ }^{7}$. For example, dewetting may appear due to differential evaporation of the solvents ${ }^{8-11}$. Other capillary phenomena may cause convection that structures the film ${ }^{12-14}$. Boundary conditions strongly affect the evaporation ${ }^{15}$, as discussed notably for evaporation of droplets ${ }^{16,17}$. Furthermore, in deposited films of colloidal dispersions cracks have been regularly observed ${ }^{18,19}$. Crack mechanism has been studied for thin films of coating ${ }^{20}$, old paintings $^{21}$ and even blood ${ }^{22}$. These processes lead to heterogeneities and may alter the properties of the material over time $^{23}$, so they need to be limited. Some solutions already exist for these described instabilities ${ }^{24-26}$.

We have discovered a new kind of instability for water-inoil emulsion drying films, deposited on a polyester plastic sheet at thickness from 20 to $150 \mu \mathrm{m}$. During water droplets evaporation, cracks appear and separate droplets into aggregates. These aggregates describe a regular pattern. We experimentally show that this pattern is exhibited only if the emulsion is adhesive, according to a characteristic length which increases with the film thickness but does not depend on volume fraction of droplets. These experimental results lead us to a phenomenological explanation.

\section{MATERIALS AND METHODS}

The studied emulsion is a water-in-oil emulsion. The internal phase consists of $\mathrm{MgSO}_{4}$ solution $(2 \% \mathrm{wgSO} 4$ (Sigma
Aldrich) in deionized water). The external phase is a mixture of dodecane (Sigma Aldrich) and silicone oil (1,0oo cSt, Aldrich), the relative proportions of which can be varied. Water droplets are stabilized with Span 8o (sorbitan monooleate, Sigma), at $0.9 \%$ w regarding the oily phase. A primary concentrated emulsion is formulated with a large quantity of surfactant $(15 \% \mathrm{w})$ and silicone oil $(8 \% \mathrm{w})$ and a small amount of dodecane $(2 \% \mathrm{w})$, adding progressively $75 \% \mathrm{w}$ of salted water mixed manually with a mortar. To reduce the surfactant concentration to $0.9 \% \mathrm{w}$, and eventually adjust the ratio of dodecane to silicone oil, this primary emulsion is diluted and centrifuged at 2oog. Water droplets are concentrated as a pellet at the bottom of the centrifuge tube, where they have volume fraction of about $75 \% \mathrm{v}$, which corresponds to $80 \% \mathrm{w}$. We get a monodisperse emulsion with small droplets. Diameter has been measured about $600 \mathrm{~nm}$ using static light scattering (Mastersizer 300o, Malvern). To enhance contrast and improve observation, tracers (iron oxide particles, diameter $300 \mathrm{~nm}$, Sensient) may be added afterwards, about $0.1 \%$ w. We have checked that they follow the droplets organization without disturbing the phenomenon.

Surface tension between the two phases of the emulsion is measured using the pendant drop method (Drop Shape Analyzer, Krüss). Contact angle between droplets is estimated observing big droplets (about 3oum diameter) of the studied emulsions under an inverted microscope (Eclipse Ti, Nikon ${ }^{27}$. Rheological characterization is done using a stress-controlled rheometer (Discovery AR-G2, TAInstruments) with stress $=6 \mathrm{mPa}$, oscillatory frequency $=$ $1.5 \mathrm{rad} / \mathrm{s}$ and strain $=2 \%$.

Emulsions are spread using an automatic film applicator (byko-drive, BYK) and a bar applicator (PA-5355, BYK) on a transparent polyester film (PA-287o, BYK). This substrate has been chosen because it is convenient to spread the 
sample and to observe the sample with a transmission microscope. Moreover, it is used in cosmetics industry because the wetting properties are close to those of the skin. The initial film thickness is measured just after application using a wet film thickness gauge (Erichsen). The uncertainty is estimated at $50 \pm 5 \mu \mathrm{m}$ considering the standard deviation for an emulsion applied on 5 different films at a given thickness. The film is observed under a transmission microscope (Eclipse Ti, Nikon) over time. Image data is processed using Fourier Transform radially averaged to quantify the patterning. Images are recorded with a $4 \mathrm{x}$ magnification to get at least 10 iterations of the pattern. The analysis is limited by the size of the image $L_{\text {image }}=3240$ $\mu \mathrm{m}$ and the size of a pixel $L_{\text {pixel }}=1.62 \mu \mathrm{m}$. A dark reference - with an opaque sample - is subtracted and the images are normalized by the illumination background which corresponds to the bare substrate. If a spatial frequency $f$ stands out in the Fourier analysis of normalized images, the patterning describes a characteristic length $L_{c a r}=1 / f$. Evaporation of emulsion layers is measured thanks to mass measurement. The system \{film + substrate $\}$ is weighed over time. Mass is deduced from initial mass and then normalized by the effective radius of the film. This enable to compare samples of different surfaces ${ }^{28}$.

\section{RESULTS AND DISCUSSION}

\section{Water evaporation leads to film patterning}

For a $50 \mu \mathrm{m}$ thick film of an emulsion containing $19.1 \% \mathrm{w}$ silicone oil, $80 \% \mathrm{w}$ of dodecane and $0,9 \% \mathrm{w}$ of surfactant in the external phase, $80 \% \mathrm{w}$ water and $0.1 \% \mathrm{w}$ tracers, the film is homogeneous just after deposition; concentrated droplets are in contact. As shown on figure 1, cracks appear after a few minutes between the droplets that become separated with oil (see Movie 1). Droplets scatter light and appear dark, whereas bright surface corresponds to oil. Droplet aggregates draw a pattern and then retract. Tracking the mass of the film over time shows that it is essentially water that evaporates from the film, while the amount of oil remains almost constant (see figure $\mathrm{S}_{1}$ ). As we never observe coalescence events between the drops, their radius must then decrease over time. Tracers follow and emphasize droplet organization that remains even after evaporation. At initial time, the Fourier Transform spectrum describes a uniform picture. Over time, a characteristic length of typically a hundred microns, corresponding to the distance between two aggregates, is exhibited. This length remains constant over time from apparition of the aggregates until complete evaporation of the internal phase (see figure 1.c).

During the whole process, the interface is glossy so no surface rugosity has appeared; the interface remains flat.

\section{Role of adhesion between droplets}

To understand this phenomenon, we have considered physico-chemical properties of emulsions, in particular droplet interaction. Previous works have shown that this kind of inverse emulsions may be adhesive ${ }^{27}$, which means there is a strong attraction between the droplets. Once in

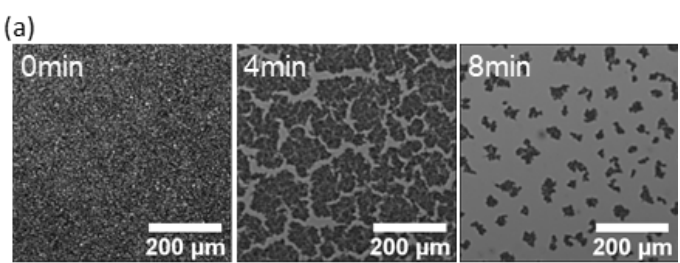

(b) Side view

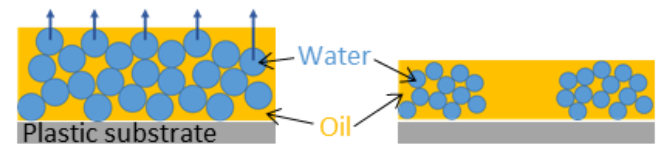

(c)

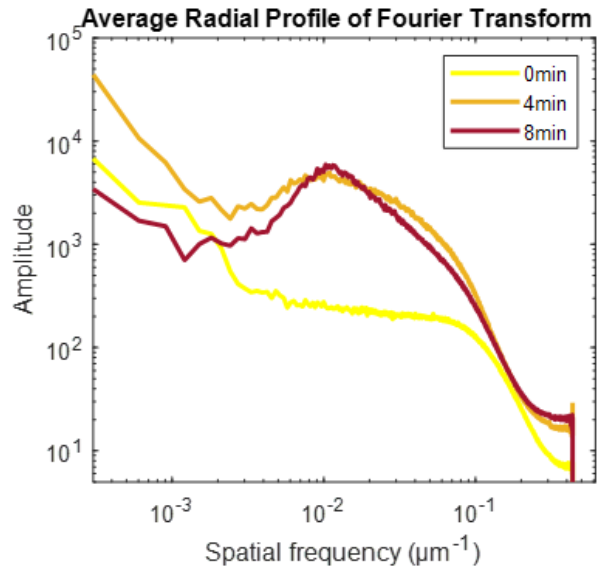

Figure 1. (a) Zoom of transmission microscope images for a $50 \mu \mathrm{m}$ thick film of emulsion containing $19.1 \% \mathrm{w}$ silicone oil, $0,9 \%$ w surfactant and $80 \% \mathrm{w}$ dodecane in external phase, $80 \%$ w water and $0.1 \% \mathrm{w}$ tracers just after application, 4 min after application and $8 \mathrm{~min}$ after application, when all water has evaporated. A regular pattern appears over time. (b) Scheme of the observed instability: during drying, water droplets evaporate, their size decreases and they create aggregates separated by oil. (c) Radially averaged Fourier Transform of microscope images: a pattern appears according to a characteristic length that remains constant over time.

contact, they are distorted according to a contact angle $\theta$ as illustrated in the inset of figure 2 . The adhesion depends on different parameters ${ }^{29}$ including surfactant solubility: the less soluble the surfactant in the external phase, the more adhesive is the emulsion ${ }^{27}$. Indeed, the surfactant is localized at the surface of the droplets. When its hydrophobic tail is in poor solvent, it tends to interact with the surfactant molecules on neighbouring droplets, and form a bilayer between the droplets. In our case, Span 80 is very soluble in dodecane but not in silicone oil, so adhesion increases with the quantity of silicone oil in the external phase $^{27}$. A range of emulsions more or less adhesive is formulated, by changing the ratio dodecane/silicone oil in the external phase but keeping the same concentration of surfactant. They all come from the same primary emulsion, only dilution is adjusted. We thus consider that they are identical, including droplet size, except for the adhesion. These emulsions are applied in thin films and observed during drying. For low amounts of silicone oil $(<1 \% w)$, no patterning is observed and no characteristic length is 
measured. For larger amounts of silicone oil ( $>1 \% \mathrm{w})$, a pattern appears with a characteristic length that increases with the quantity of silicone oil, as shown on figure 2 .

To determine whether the studied emulsions are adhesive or not, the elastic modulus is measured for each of them,

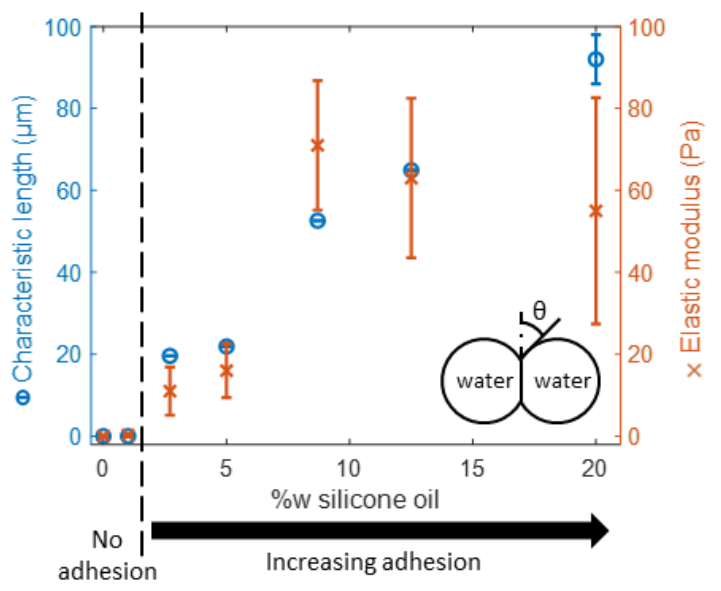

Figure 2. The characteristic length (blue circles) is measured for a $50 \mu \mathrm{m}$ thick film of emulsions containing various concentrations of silicone oil and dodecane in external phase, $80 \% \mathrm{w}$ water and $0.1 \%$ w tracers, after drying. The uncertainty is estimated to $6 \mu \mathrm{m}$ thanks to the standard deviation on a given emulsion applied on five different films. Elastic modulus (orange crosses) is measured for emulsions containing various concentrations of silicone oil, $63 \% \mathrm{w}$ of water and $0.1 \% \mathrm{w}$ tracers, with oscillating shear at $1.5 \mathrm{rad} / \mathrm{s}$, stress $6 \mathrm{mPa}$, strain $2 \%$. Error bars are estimated to $30 \%$ according variation coefficient of 5 repetitions of the measurement on a given emulsion (19.1\%w silicone oil, 80\%w dodecane and $0.9 \% \mathrm{w}$ surfactant in external phase, $63 \% \mathrm{w}$ water and $0.1 \% \mathrm{w}$ tracers). Adhesion increases with the concentration of silicone oil in external phase. Patterning appears when the emulsion is adhesive. Inset: schematic of two adhesive droplets.

slightly diluted at $63 \% \mathrm{w}$, corresponding to $56 \% \mathrm{v}$ of water. Indeed, it has been shown that adhesive emulsions exhibit an elastic modulus even below random close packing $(\approx$ $64 \% \mathrm{v}$ ): for an adhesive emulsion, the bonds between droplets leads to the formation of a cohesive connected network of aggregates and create a gel ${ }^{\circ}$ that can support a shear stress $^{31}$. As shown on figure 2, emulsions containing less than $1 \% \mathrm{w}$ of silicone oil in external phase exhibit no elasticity, so they are not adhesive. On the contrary, the ones containing more than $1 \% \mathrm{w}$ of silicone oil demonstrate significant elasticity, so they are adhesive. These results are consistent with microscopic observation when emulsions are diluted: for non-adhesive emulsions, droplets are separated and present a clear Brownian motion whereas as silicon oil amount increases above $1 \% \mathrm{w}$, droplets aggregate. We conclude that patterning occurs only for adhesive emulsions and the characteristic length increases with silicone oil amount, which means with adhesion.

\section{Patterning depends on film thickness}

The behavior of an adhesive emulsion (19.1\%w silicone oil and $80 \%$ w dodecane in external phase, $80 \% \mathrm{w}$ water and
$0.1 \% \mathrm{w}$ tracers) has been investigated at different film thicknesses and volume fractions of water. A pattern appears whatever the thickness in the studied range, from 20 to 150 $\mu \mathrm{m}$, and concentration of water droplets, from $25 \% \mathrm{w}$ to $80 \% \mathrm{w}$ corresponding to volume fractions from $20 \% \mathrm{v}$ to $75 \% \mathrm{v}$. The characteristic length increases linearly with the initial thickness, as shown on figure 3. However, we show that it does not depend on volume fraction of droplets (see figure $\mathrm{S}_{2}$ ). This result is surprising because volume fraction

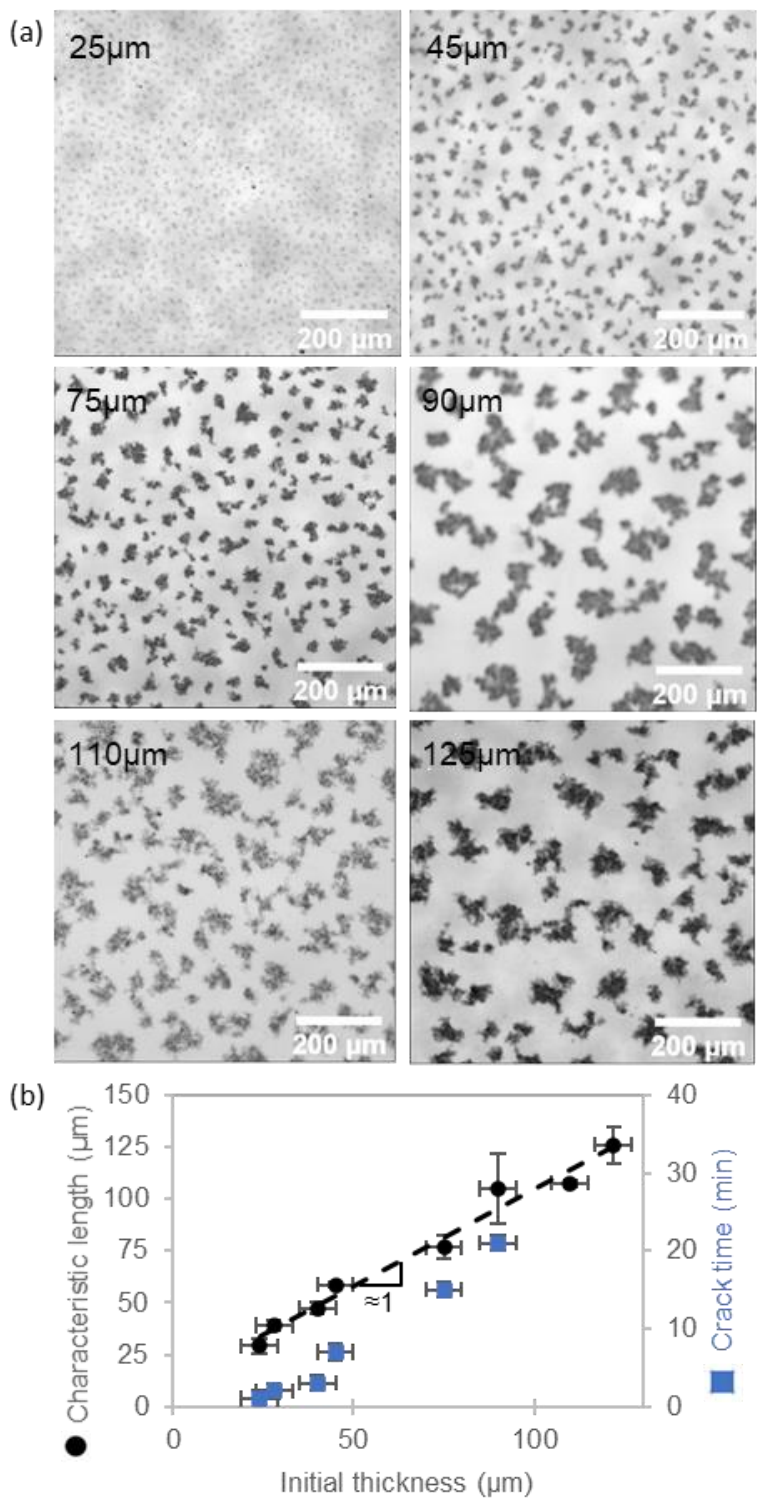

Figure 3. Characteristic length increases with the initial thickness. (a) Zoom of transmission microscope images for an adhesive emulsion $(19.1 \% \mathrm{w}$ silicone oil and $80 \% \mathrm{w}$ dodecane in external phase) with $80 \% \mathrm{w}$ water and $0.1 \% \mathrm{w}$ tracers applied on plastic sheet at different initial thicknesses, after drying. (b) Characteristic length measured after drying (black dots) and time of crack (blue squares) according to initial thickness for this adhesive emulsion. $L_{c a r}$ data corresponds to the mean value \pm standard deviation calculated based on measurements on 5 different locations of each film. The dotted line corresponds to the linear fit of eq. 2. 
of droplets influences the rheological properties of the emulsion, and we could expect that rheology has a strong impact on a kinetic phenomenon like this one.

The kinetics of the cracks can be followed binarizing the images and quantifying the bright surface (see figure $S_{3}$ ), that is the surface where this is no droplets. Just after deposition, the image is mainly dark because the scattering droplets are concentrated and all connected. When cracks appear, bright surface increases according to shrinkage speed. When all water has evaporated, almost the entire picture is bright, only tracers remain. We can extract the time of crack, shown on figure 3 for different initial thicknesses, with a relative uncertainty of few minutes depending on the binarization threshold. Evaporation measurement show that in the first minutes, evaporation does not depend on thickness of the film and corresponds to evaporation of bulk water (see figure $\mathrm{S}_{1}$ ). Considering the time of crack and the evaporation rate, we compute that cracks appear when droplets volume has decreased of $\delta \approx 10 \%$, whatever the initial thickness is. The rupture seems to be due to geometrical constrains on the droplets: they have retracted of a given volume at crack time.

\section{Proposed model for drying emulsion patterning}

Numerous instabilities have been observed in drying films: changes in composition may induce dewetting ${ }^{9}$, convection ${ }^{13}$, phase transition ${ }^{32}, \ldots$ In our case, the instability takes place on oleophilic (Polyester) or hydrophilic (glass, data not shown) solid surfaces, which seems to exclude dewetting phenomena. The air-liquid interface remains flat throughout water evaporation, which is not compatible with the appearance of Marangoni type instability coupled to hydrodynamics. Finally, the composition of the continuous phase remains essentially unchanged during the experiment, and the phenomenon is independent of the volume fraction of the water drops, which presumably rules out a phase transition in the oil, or of the emulsion as a whole.

Observed patterns may also be due to mechanical instabilities, when mechanical stress is released through cracks or delamination. Crack mechanism has been studied for thin films of coating ${ }^{20}$, paints ${ }^{21}$ or miscellaneous colloidal dispersions ${ }^{33}$. Upon solvent evaporation, films bounded to substrate often develop tensile stresses. Opening cracks are considered when the elastic energy stored in the material becomes greater than the interfacial energy needed to create new surfaces.

One key observation in our case is that the instability appears only for adhesive emulsions, i.e. for samples with a non-zero elastic modulus. We will therefore consider here a mechanical model of the instability. For an adhesive emulsion, the bonds between droplets leads to the formation of a connected network of aggregates and create a gel $^{30}$ that can support a shear stress ${ }^{3}$. We will assume that this gel is also connected to the plastic film underneath. Indeed, in the case of adhesive inverse emulsions, at the surface of the droplets, the hydrophobic part of the surfactant being in poor solvent, it generally prefers to adhere to hydrophobic substrates (or to form bilayers between the droplets, or between droplets and hydrophilic substrates). Shrinkage of water droplets during water evaporation creates elastic stress among the gel, that cannot be relaxed due to the adhesion to the substrate. At some point, it has to be released through a crack. In accordance to this hypothesis, no significant movement is observed before the appearance of fractures (Fig. 1 and movie in SI).

More precisely, we will model the emulsion as an isotropic elastic film bounded to a rigid substrate. The film is uniformly stressed prior cracking. A simplified two-dimensional model has been proposed for such cases, which accounts for multiple experimental observations ${ }^{34,35}$. When a linear fracture appears, the stress will relax transversally on each side of the fracture, over a distance of the order of the thickness $h$, with an exponential decay ${ }^{34}$. At larger distances, adhesion to the rigid substrate prevents stress relaxation, so new fractures may appear. In the case of parallel cracks, the distance between the fractures $L_{c a r}$ should be then around $2^{*} h$. A more rigorous calculation shows indeed that the characteristic length $L_{c a r}$ between two parallel fractures is proportional to the thickness $h$, with a numerical prefactor that depends on the elastic response of the material 35 .

We can see that this simple mechanical model accounts for our two major experimental observations: on the one hand, instability does not appear for non-adhesive emulsions (Fig. 2), because there can be no tension stress in the emulsion. On the other hand, the characteristic length of the instability is proportional to the thickness (Fig.3). It is interesting to note that this model, originally used for rigid materials (elastic modulus $>\mathrm{MPa}$ ), applies equally well to these soft materials (elastic modulus of a few tens of Pa).

If we compare to mechanical instability with suspension of solid particles, we can note several differences. The origin of the instability is different in that case, it is related to the capillary action. The meniscus between particles at the interface with air reduces the pressure inside the film, and thus induces the tensile stress. The properties of the dispersed phase will strongly influence the process (initial volume fraction of particles, their deformability) ${ }^{21}$. In our case, it is rather the properties of the continuous phase that are important (solubility of the surfactant). These findings are important because they imply very different solutions if we want to avoid fracture formation.

\section{CONCLUSION}

We describe a new instability in drying emulsion films occurring during evaporation of the internal phase: cracks appear between adhesive droplets creating aggregates according to a regular pattern. We have checked that this phenomenon is generic for different adhesive emulsions on different substrates. Considering previous works on painting cracks, we suggest that this phenomenon is due to the gel structure of this kind of emulsions and their elastic properties.

Previous works have mainly focused on effect of evaporation of the external phase. We believe that it is relevant to 
deepen our results since inverse emulsions are usually adhesive and widely used in industry. Furthermore, industrial systems are often even more complex, containing also colloidal particles. It will be interesting to understand the coupling effects of adhesive emulsions and colloidal dispersions.

\section{ASSOCIATED CONTENT}

Supporting Information. Complementary experimental results about evaporation, impact of the volume fraction on characteristic length and kinetics. This material is available free of charge via the Internet at http://pubs.acs.org.

\section{AUTHOR INFORMATION}

\section{Corresponding Author}

* jean.baudry@espci.psl.eu

ESPCI Paris - Laboratoire Colloïdes et Matériaux Divisés, 10 rue Vauquelin, 75 oo5 Paris, France

\section{Author Contributions}

The manuscript was written through contributions of all authors. All authors have given approval to the final version of the manuscript.

\section{Funding Sources}

This work has been supported by CHANEL Parfums Beauté and ARNT, as part as CIFRE PhD thesis. This work has also received the support of "Institut Pierre-Gilles de Gennes" (laboratoire d'excellence, "Investissements d'avenir" program ANR-10-IDEX-0oo1-02 PSL, ANR-10-LABX-31 and ANR-10EQPX-34).

\section{REFERENCES}

(1) Rickerby, D. S. A Review of the Methods for the Measurement of Coating-Substrate Adhesion. Surface and Coatings Technology 1988, $36 \quad$ (1), 541-557. https://doi.org/10.1016/0257-8972(88)90181-8.

(2) Fichot, J.; Heyd, R.; Josserand, C.; Chourpa, I.; Gombart, E.; Tranchant, J.-F.; Saboungi, M.-L. Patterned Surfaces in the Drying of Films Composed of Water, Polymer, and Alcohol. Physical Review E 2012, $86 \quad$ (6) https://doi.org/10.1103/PhysRevE.86.061601.

(3) Kim, M.; Kim, D.-J.; Ha, D.; Kim, T. Cracking-Assisted Fabrication of Nanoscale Patterns for Micro/Nanotechnological Applications. Nanoscale 2016, 8 (18), 9461-9479. https://doi.org/10.1039/C5NR06266G.

(4) Simões, M.; Assis, O. B. G.; Avaca, L. A. Some Properties of Protective Sol-Gel Glass Coatings on Sintered Stainless Steels. Journal of Non-Crystalline Solids 2000, 273 (1), 159163. https://doi.org/10.1016/S0022-3093(00)00161-7.

(5) Arikawa, H.; Kanie, T.; Fujii, K.; Ban, S.; Homma, T.; Takahashi, H. Optical and Color Stabilities of Paint-on Resins for Shade Modification of Restorative Resins. Dental Materials Journal 2004, 23 (2), 155-160. https://doi.org/10.4012/dmj.23.155.

(6) Gilbert, L.; Savary, G.; Grisel, M.; Picard, C. Predicting Sensory Texture Properties of Cosmetic Emulsions by Physical Measurements. Chemometrics and Intelligent Laboratory Systems 2013, 124, 21-31. https://doi.org/10.1016/j.chemolab.2013.03.002.

(7) Zhou, J.; Man, X.; Jiang, Y.; Doi, M. Structure Formation in Soft-Matter Solutions Induced by Solvent Evaporation. $A d-$ vanced Materials 2017, $29 \quad$ (45), 1703769. https://doi.org/10.1002/adma.201703769.
Schwartz, L. W.; Roy, R. V.; Eley, R. R.; Petrash, S. Dewetting Patterns in a Drying Liquid Film. Journal of Colloid and Interface Science 2001, 234 (2), 363-374. https://doi.org/10.1006/jcis.2000.7312.

(9) Reiter, G. Dewetting of Thin Polymer Films. Phys. Rev. Lett. 1992, 68 (1), 75-78 https://doi.org/10.1103/PhysRevLett.68.75.

(10) Torun, N.; Torun, I.; Sakir, M.; Kalay, M.; Onses, M. S. Physically Unclonable Surfaces via Dewetting of Polymer Thin Films. ACS Appl. Mater. Interfaces 2021, 13 (9), 1124711259. https://doi.org/10.1021/acsami.0c16846.

(11) Janiszewska, N.; Raczkowska, J.; Budkowski, A.; Gajos, K.; Stetsyshyn, Y.; Michalik, M.; Awsiuk, K. Dewetting of Polymer Films Controlled by Protein Adsorption. Langmuir 2020, 36 (40), 11817-11828. https://doi.org/10.1021/acs.langmuir.0c01718.

(12) Bassou, N.; Rharbi, Y. Role of Bénard-Marangoni Instabilities during Solvent Evaporation in Polymer Surface Corrugations. Langmuir 2009, 25 (1), 624-632. https://doi.org/10.1021/la802979a.

(13) Sobac, B.; Colinet, P.; Pauchard, L. Influence of Bénard-Marangoni Instability on the Morphology of Drying Colloidal Films. Soft Matter 2019, 15 (11), 2381-2390. https://doi.org/10.1039/C8SM02494D.

(14) Tree, D. R.; Iwama, T.; Delaney, K. T.; Lee, J.; Fredrickson, G. H. Marangoni Flows during Nonsolvent Induced Phase Separation. ACS Macro Lett. 2018, 7 (5), 582-586. https://doi.org/10.1021/acsmacrolett.8b00012.

(15) Wang, F.; Wu, M.; Man, X.; Yuan, Q. Formation of Deposition Patterns Induced by the Evaporation of the Restricted Liquid. Langmuir 2020, 36 (29), 8520-8526. https://doi.org/10.1021/acs.langmuir.0c01116.

(16) Larson, R. G. Twenty Years of Drying Droplets. Nature 2017, 550 (7677), 466-467. https://doi.org/10.1038/550466a.

(17) Zang, D.; Tarafdar, S.; Tarasevich, Y. Yu.; Dutta Choudhury, M.; Dutta, T. Evaporation of a Droplet: From Physics to Applications. Physics Reports 2019, 804, 1-56. https://doi.org/10.1016/j.physrep.2019.01.008.

(18) Singh, K. B.; Tirumkudulu, M. S. Cracking in Drying Colloidal Films. Phys. Rev. Lett. 2007, 98 (21), 218302. https://doi.org/10.1103/PhysRevLett.98.218302.

(19) Routh, A. F. Drying of Thin Colloidal Films. Rep. Prog. Phys. 2013, 76 (4), 046603. https://doi.org/10.1088/00344885/76/4/046603.

(20) Atkinson, A.; Guppy, R. M. Mechanical Stability of Sol-Gel Films. $J$ Mater Sci 1991, 26 (14), 3869-3873 https://doi.org/10.1007/BF01184984.

(21) Giorgiutti-Dauphiné, F.; Pauchard, L. Painting Cracks: A Way to Investigate the Pictorial Matter. Journal of Applied $\begin{array}{lllll}\text { Physics } & \text { 2016, } & 120 & \text { (6), } & 065107 .\end{array}$ https://doi.org/10.1063/1.4960438.

(22) Iqbal, R.; Shen, A. Q.; Sen, A. K. Understanding of the Role of Dilution on Evaporative Deposition Patterns of Blood Droplets over Hydrophilic and Hydrophobic Substrates. Journal of Colloid and Interface Science 2020, 579, 541-550. https://doi.org/10.1016/j.jcis.2020.04.109.

(23) Binks, B. P.; Fletcher, P. D. I.; Johnson, A. J.; Marinopoulos, I.; Crowther, J. M.; Thompson, M. A. Evaporation of ParticleStabilized Emulsion Sunscreen Films. ACS Applied Materials \& Interfaces 2016, 8 (33), 21201-21213. https://doi.org/10.1021/acsami.6b06310.

(24) Boulogne, F.; Pauchard, L.; Giorgiutti-Dauphiné, F. Effect of a Non-Volatile Cosolvent on Crack Patterns Induced by Desiccation of a Colloidal Gel. Soft Matter 2012, 8 (32), 85058510. https://doi.org/10.1039/C2SM25663K.

(25) Kaewpetch, T.; Gilchrist, J. F. Chemical vs. Mechanical Microstructure Evolution in Drying Colloid and Polymer Coatings. Scientific Reports 2020, $10 \quad$ (1), 10264. https://doi.org/10.1038/s41598-020-66875-0.

(26) Fischer, S. B.; Koos, E. Using an Added Liquid to Suppress Drying Defects in Hard Particle Coatings. Journal of Colloid 
and Interface Science 2021, 582, 1231-1242. https://doi.org/10.1016/j.jcis.2020.08.055.

(27) Poulin, P.; Bibette, J. Adhesion of Water Droplets in Organic Solvent. Langmuir 1998, 14 (22), 6341-6343. https://doi.org/10.1021/la9801413.

(28) Dollet, B.; Boulogne, F. Natural Convection above Circular Disks of Evaporating Liquids. Physical Review Fluids 2017, 2 (5). https://doi.org/10.1103/PhysRevFluids.2.053501.

(29) Poulin, P.; Bibette, J. Wetting of Emulsions Droplets: From Macroscopic to Colloidal Scale. Phys. Rev. Lett. 1997, 79 (17), 3290-3293. https://doi.org/10.1103/PhysRevLett.79.3290.

(30) Bibette, J.; Mason, T. G.; Gang, H.; Weitz, D. A.; Poulin, P. Structure of Adhesive Emulsions. Langmuir 1993, 9 (12), 3352-3356. https://doi.org/10.1021/la00036a006.

(31) Datta, S. S.; Gerrard, D. D.; Rhodes, T. S.; Mason, T. G.; Weitz, D. A. Rheology of Attractive Emulsions. Phys. Rev. E
2011 ,

84

(4),

041404.

https://doi.org/10.1103/PhysRevE.84.041404. ral Development of Nanoparticle Dispersion during Drying in Polymer Nanocomposite Films. Macromolecules 2016, 49 (23), 9068-9079. https://doi.org/10.1021/acs.macromol.6b01939.

(33) Groisman, A.; Kaplan, E. An Experimental Study of Cracking Induced by Desiccation. EPL 1994, 25 (6), 415-420 https://doi.org/10.1209/0295-5075/25/6/004

(34) Xia, Z. C.; Hutchinson, J. W. Crack Patterns in Thin Films. Journal of the Mechanics and Physics of Solids 2000, 48 (6), 1107-1131. https://doi.org/10.1016/S0022-5096(99)00081-2.

(35) Tirumkudulu, M. S.; Russel, W. B. Cracking in Drying Latex Films. Langmuir 2005, 21 (11), 4938-4948. https://doi.org/10.1021/la048298k. 
TOC GRAPHIC

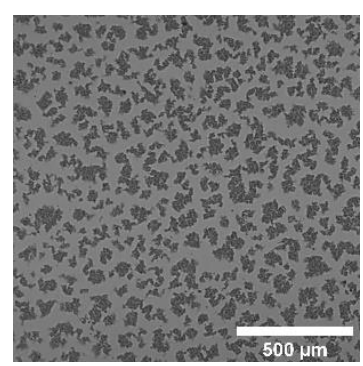

